\title{
The next generation HAPPEX experiments
}

\author{
Richard S. Holmes, for the HAPPEX collaboration \\ Syracuse University, Syracuse, NY 13244, USA \\ Received: 15 October 2004 / Published Online: 8 February 2005 \\ (C) Società Italiana di Fisica / Springer-Verlag 2005
}

\begin{abstract}
A new generation of parity violating electron scattering experiments began running in Summer 2004 at the Thomas Jefferson National Accelerator Facility. HAPPEX-H will measure the parity violating asymmetry in polarized electron scattering from protons at $Q^{2}=0.11(\mathrm{GeV} / \mathrm{c})^{2}$. This asymmetry is sensitive to a linear combination of the strange electric and magnetic form factors of the proton. HAPPEX-He will measure the parity violating asymmetry in polarized electron scattering from a ${ }^{4} \mathrm{He}$ target at the same value of $Q^{2}$, using the same spectrometers and similar detectors, accessing the strange electric form factor cleanly. PREX will use parity violating electron scattering to determine the neutron radius of the ${ }^{206} \mathrm{~Pb}$ nucleus.

PACS. 13.60.Fz Elastic and Compton scattering - 11.30.Er Charge conjugation, parity, time reversal, and other discrete symmetries - 13.40.Gp Electromagnetic form factors - 14.20.Dh Protons and neutrons - 24.80.+y Nuclear tests of fundamental interactions and symmetries - 21.10.Gv Mass and neutron distributions - 25.30.Bf Elastic electron scattering
\end{abstract}

\section{Introduction}

The question of how sea quarks contribute to nucleon observables has been of great interest since the European Muon Collaboration's observation [1] that valence quarks contribute less than half of the proton spin. Kaplan and Manohar 2] suggested that strange quarks (necessarily sea quarks) could contribute to the vector matrix elements of the nucleon, giving rise to nonzero strange electric and magnetic form factors.

The size of these effects can be deduced from measurements of the parity-violating asymmetry in elastic scattering of longitudinally polarized electrons from protons or nuclei [3, 4, 5]. These asymmetries are functions of the conventional electromagnetic form factors of the nucleons, an axial form factor, and the strange form factors. Asymmetries measured in three such experiments $6,7,8,9]$ have been reported.

Polarized electron scattering from a high-Z nucleus can be used to address very different physics. The parity violating asymmetry measured in such an experiment is sensitive to the neutron radius of the target nucleus [10, an observable which has to date not been measured in a clean, model independent way.

I report here on a set of second-generation parity violating electron scattering experiments which will be performed at the Thomas Jefferson National Accelerator Facility (Jlab). They will provide improved sensitivity to the strange form factors at low $Q^{2}$, and a clean measurement of the neutron radius of the ${ }^{206} \mathrm{~Pb}$ nucleus.

\section{Experimental goals}

The parity violating asymmetry in scattering of polarized electrons from protons is given by

$$
\begin{aligned}
A^{\mathrm{PV}}= & -\frac{G_{F}|Q|^{2}}{4 \pi \alpha \sqrt{2}} \times \rho^{\prime}\left[\left(1-4 \kappa^{\prime} \sin ^{2} \theta_{W}\right)-\right. \\
& \left.\frac{\epsilon G_{E}^{\gamma p}\left(G_{E}^{\gamma n}+G_{E}^{s}\right)+\tau G_{M}^{\gamma p}\left(G_{M}^{\gamma n}+G_{M}^{s}\right)}{\epsilon\left(G_{E}^{\gamma p}\right)^{2}+\tau\left(G_{M}^{\gamma p}\right)^{2}}\right] \\
& +A_{A}
\end{aligned}
$$

where $G_{E(M)}^{\gamma p}$ is the electric (magnetic) Sachs form factor for the proton, $G_{E(M)}^{\gamma n}$ is the same for the neutron, $G_{E}^{s}$ and $G_{M}^{s}$ are the electric and magnetic strange form factors, $\theta_{W}$ is the weak mixing angle, $G_{F}$ is the Fermi constant, and $\alpha$ is the fine-structure constant. $A_{A}$ is a term proportional to $G_{A}^{Z p}$, the neutral weak axial form factor. The kinematic factors are $Q^{2}=-q_{\mu}^{2}>0$, the square of the four-vector momentum transfer, $\tau=Q^{2} / 4 M^{2}$ where $M$ is the proton mass, and $\epsilon=\left[1+2(1+\tau) \tan ^{2}(\theta / 2)\right]^{-1}$ where $\theta$ is the scattering angle. Finally, $\rho^{\prime}=0.9879$ and $\kappa^{\prime}=1.0029$ are parameters arising from electroweak radiative corrections [11]. At forward angles $A_{A}$ is small, and a linear combination of the strange form factors can be calculated from the asymmetry and measured values of the nucleon electromagnetic form factors. One can express a strangeness radius $\rho_{s}$ and a strange magnetic moment $\mu_{s}$ in terms of the strange form factors as $\rho_{s}=\left[d G_{E}^{s} / d \tau\right]_{\tau=0}$, $\mu_{s}=G_{M}^{s}(0)$. 
The original Hall A Proton Parity Experiment (HAPPEX) measured parity violating asymmetry in the elastic scattering of polarized electrons from a liquid hydrogen target at $\theta=12.3$ degrees, $Q^{2}=0.5(\mathrm{GeV} / \mathrm{c})^{2}$. The result [7] was $A^{\mathrm{PV}}=-14.92 \pm 0.98$ (stat) \pm 0.56 (syst) parts per million (ppm); from this we obtain $G_{E}^{s}+0.392 G_{M}^{s}=$ $0.014 \pm 0.020 \pm 0.010$ where the first error is experimental and the second is due to errors in the values of the electromagnetic form factors. The strange form factors may be small at this $Q^{2}$, or there may be an accidental cancellation. It is of interest to measure $G_{E}^{s}$ and $G_{M}^{s}$ separately, and particularly at a smaller value of $Q^{2}$ to better allow extrapolation to zero. These considerations have motivated two new experiments, HAPPEX-H (Experiment E99-115) [12] and HAPPEX-He (Experiment E00-114) [13].

HAPPEX-H, like HAPPEX, scatters polarized electrons elastically from a hydrogen target. However, with the use of the new septum magnet upgrades to the HRS spectrometers the average laboratory scattering angle is reduced to approximately 6 degrees. The $Q^{2}$ for this experiment is about $0.1 \mathrm{GeV}^{2}$ and the theoretical value for the parity violating asymmetry in the absence of strange quark effects is $1.6 \mathrm{ppm}$. The goal is to measure this asymmetry with statistical and systematic errors of $5 \%$ and $2.5 \%$, respectively.

Obtaining values for the two strange form factors separately requires a measurement at different kinematics or on a different target. A particularly attractive choice is to use a spinless isoscalar target in which case the parity violating asymmetry for elastic scattering of polarized electrons is simply

$$
A^{\mathrm{PV}}=-\frac{A_{0}}{2}\left(2 \sin ^{2} \theta_{W}+\frac{G_{E}^{s}}{G_{E}^{\gamma p}+G_{E}^{\gamma n}}\right)
$$

and $A^{\mathrm{PV}}$ is sensitive only to $G_{E}^{s}$. HAPPEX-He scatters polarized electrons from a high pressure ${ }^{4} \mathrm{He}$ target with the same kinematics as HAPPEX-H: $\theta \sim 6$ degrees, $Q^{2} \sim 0.1$ $\mathrm{GeV}^{2}$. Without strange quark effects, the predicted asymmetry is $8.4 \mathrm{ppm}$, and the goal is to measure this asymmetry to $2.2 \%$ statistical and $2.1 \%$ systematic. Both HAPPEX-H and HAPPEX-He began data taking in Summer 2004.

The parity violating asymmetry in elastic scattering of polarized electrons from a heavy nucleus is sensitive to the ratio $R_{n} / R_{p}$ of the neutron and proton radii for that nucleus. The lead radius experiment, PREX (Experiment E00-114) 14, will measure this asymmetry with a ${ }^{208} \mathrm{~Pb}$ target. Kinematics will be $\theta \sim 6$ degrees, $Q^{2} \sim 0.01 \mathrm{GeV}^{2}$, with an expected asymmetry $A^{\mathrm{PV}} \sim 0.5 \mathrm{ppm}$. Our goal is to measure this asymmetry with statistical and systematic errors of $3 \%$ and $1.1 \%$, respectively, yielding a $1 \%$ measurement of $R_{n} / R_{p}$. PREX is conditionally approved.

A schematic of the HAPPEX-H experiment is shown in Fig. 1, Polarized electrons are produced at the source using circularly polarized light incident on a GaAs crystal. Polarization is set at $30 \mathrm{~Hz}$, with a randomly chosen polarization followed by its complement to make a pair of opposite helicity "windows" each about $30 \mathrm{~ms}$ in length.

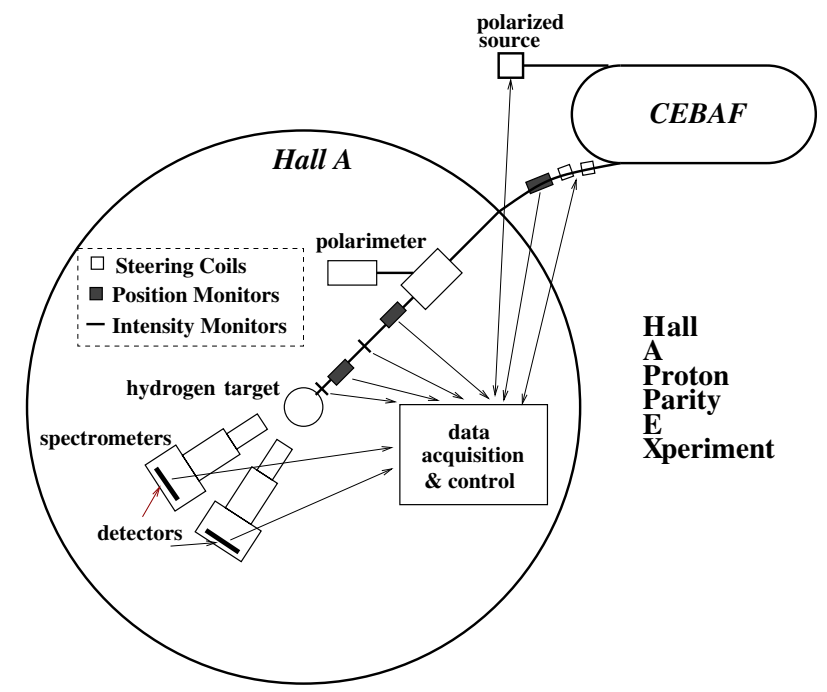

Fig. 1. Schematic of HAPPEX-H experiment

In Hall A, elastically scattered electrons are focused onto two detectors by two identical magnetic spectrometers. Inelastic events are cleanly rejected by being focused out of the detector acceptance.

The high rate in the detectors would result in significant dead time issues for a counting experiment. Instead, we integrate the detector signals over each helicity window using custom built integrating ADCs and a data acquisition system triggered at $30 \mathrm{~Hz}$. The beam position, energy, and intensity are measured using various detectors in the Hall A beam line and these signals are also integrated over each helicity window.

The physics asymmetry is given by

$$
A_{\text {phys }} \sim A_{d e t}-A_{Q}+\alpha A_{E}+\sum i \beta_{i} \Delta x_{i}
$$

Here $A_{\text {det }}=\left(S^{R}-S^{L}\right) /\left(S^{R}+S^{L}\right)$ is the helicity correlated asymmetry in the integrated detector signals $S^{R(L)}$ for right (left) polarized beam. The remaining terms are corrections for systematic effects. $A_{Q}$ and $A_{E}$ are the helicity correlated relative differences in the beam intensity and energy, respectively. $\Delta x_{i}$ is the helicity correlated difference in beam parameter $x_{i}$, where the four parameters are horizontal and vertical positions and angles at the target. $\alpha$ and $\beta_{i}$ are the responses of the detector to changes in beam energy and position or angle $x_{i}$, respectively. This equation is correct under the assumptions that the detectors and beam monitors are linear and that there are no additional sensitivities to higher order parameters, such as spot size.

\section{Beam and instrumentation upgrades}

In order to meet the challenging goals of these experiments, we must control and understand our systematics at a more precise level than in the past. A number of upgrades and improvements to the polarized source, beam transport, and experimental instrumentation are required, 
Table 1. HAPPEX beam requirements. "Jitter" is RMS width for signals integrated over $30 \mathrm{~ms}$; "Difference" is size of helicity correlated difference averaged over the data set

\begin{tabular}{llll}
\hline Property & Nominal & Jitter & Difference \\
\hline Energy & $3.2 \mathrm{GeV}$ & $<80 \mathrm{ppm}$ & $<13 \mathrm{ppb}$ \\
Current & $100 \mu \mathrm{A}$ & $<1000 \mathrm{ppm}$ & $<600 \mathrm{ppb}$ \\
Position & 0 & $<12 \mu \mathrm{m}$ & $<2 \mathrm{~nm}$ \\
Angle & 0 & $<12 \mu \mathrm{rad}$ & $<2 \mathrm{nrad}$ \\
Halo @ $3 \mathrm{~mm}$ & $<100 \mathrm{~Hz} / \mu \mathrm{A}$ & & \\
\hline
\end{tabular}

including: new target cells; various improvements at the polarized source; better polarimetry; new Cerenkov detectors; new luminosity monitors; new profile scanners; new beam intensity and position monitors; and an improved data acquisition system.

The correction terms in 3 must be kept small and precisely measured. Potentially the largest correction is $A_{Q}$, which can easily reach levels of several hundred ppm or larger. A standard technique for reducing this term is to introduce a feedback system, by which the helicity correlated beam intensity difference measured in the experimental hall is used to govern modulation of the intensity of the laser light at the source cathode, driving $A_{Q}$ to a value consistent with zero.

While it is possible to feed back on beam energy, position, and angle differences, correlations between these parameters and fluctuations in their measurement introduce complications best avoided if possible. Careful control of position and angle of the laser spot on the source cathode can keep these beam parameter differences small; then, by measuring the differences in the hall and calibrating the detector responses to parameter differences by introducing deliberate modulations of the beam during a portion of the data taking, the required small corrections can be made with good precision.

The revamped laser table setup at the polarized source features two complementary systems for modulating the laser intensity. A PZT mirror is available for modulating position of the laser beam, if required. As in HAPPEX there is an insertable half wave plate to provide slow helicity reversal, which suppresses certain systematics; there also is a rotatable half wave plate for control of position and intensity systematics. A lens is used to image the beam onto the photocathode. The cathode provides better than $80 \%$ polarization with intensities up to $100 \mu \mathrm{A}$.

Some of the requirements for HAPPEX-H and HAPPEX-He quality beam are shown in Table 1. Thanks to hard work by the source group in collaboration with HAPPEX personnel, these requirements seem to be achievable now. PREX requirements will be even more stringent. For further details on control of source systematics see Cates's talk at this conference.

A significant portion of the final systematic error comes from polarimetry. Two polarimeters are available in Hall A, a Møller polarimeter 15:16 and a Compton polarimeter [17,15]. For the Møller polarimeter the main uncertainty is the foil polarization; at the HAPPEX-H and HAPPEX-He energy of $\sim 3 \mathrm{GeV}$, a total relative error of 3 to $3.4 \%$ is expected.

The Compton polarimeter, which was commissioned during the first HAPPEX experiment, has since been upgraded with the addition of an electron recoil detector. In this new configuration total relative errors of $1.4 \%$ per 1 hour measurement have been seen with a $4.5 \mathrm{GeV}$ beam, and $2.0 \%$ is probably achievable at $3 \mathrm{GeV}$.

Additional improvements to polarimetry will be required for PREX, where the combination of high beam intensity and low energy $(850 \mathrm{MeV})$ means neither of the existing polarimeters can obtain precise measurements under running conditions. To address this the Compton polarimeter will be upgraded with a green laser that should enable measurements at the $1 \%$ level in under a day.

The HAPPEX-H detectors are brass/quartz sandwich Cerenkov total absorption detectors. Elastically scattered electrons are contained in a $\sim 100 \mathrm{~cm} \times 600 \mathrm{~cm}$ region of the focal plane in each spectrometer arm. To cover this region with minimal transmission losses, each detector consists of two segments at right angles, oriented to put the Cerenkov peak in line with the quartz layers and aimed at the single photomultiplier tube coupled to each segment. For HAPPEX-He, where the elastic peak region is smaller in size, we disassemble one of these detectors into its two segments and use one segment in each spectrometer arm. Detector design for PREX, where the elastic peak region will be very small, has not been finalized.

Interpretation of our results requires precise knowledge of our effective $Q^{2}$. For HAPPEX this was done using the standard Hall A drift chamber package at low beam intensity. For the new experiments we supplement this method with a pair of quartz Cerenkov profile detectors which can be scanned across the elastic peak at high beam intensity. This enables us to verify the drift chamber results and to monitor the $Q^{2}$ stability during the experiment.

The stripline position monitors used for HAPPEX have a resolution of $\sim 1.8 \mu \mathrm{m}$. This is adequate for HAPPEX-H and HAPPEX-He, but the stringent limits required for PREX demand better instrumentation. We have installed new cavity based position and intensity monitors which will be commissioned during the HAPPEX-H/HAPPEX-He running.

Target density fluctuations give rise to increased widths and correlations in the detector signals. New luminosity monitors mounted on the downstream beamline at very small scattering angles enable us to monitor such target density fluctuations. They also provide high sensitivity to helicity correlated beam parameters and enable better measurements of electronic noise. The monitors have been tested to $\sim 200 \mathrm{ppm}$ resolution per $30 \mathrm{~ms}$ helicity window at low beam intensity, and performance of $\sim 100 \mathrm{ppm}$ at high intensity is anticipated.

A new target cell design is being commissioned for HAPPEX-H and HAPPEX-He. Tests in early 2004 demonstrated that the existing design suffered from density fluctuations in a high power beam which would have 


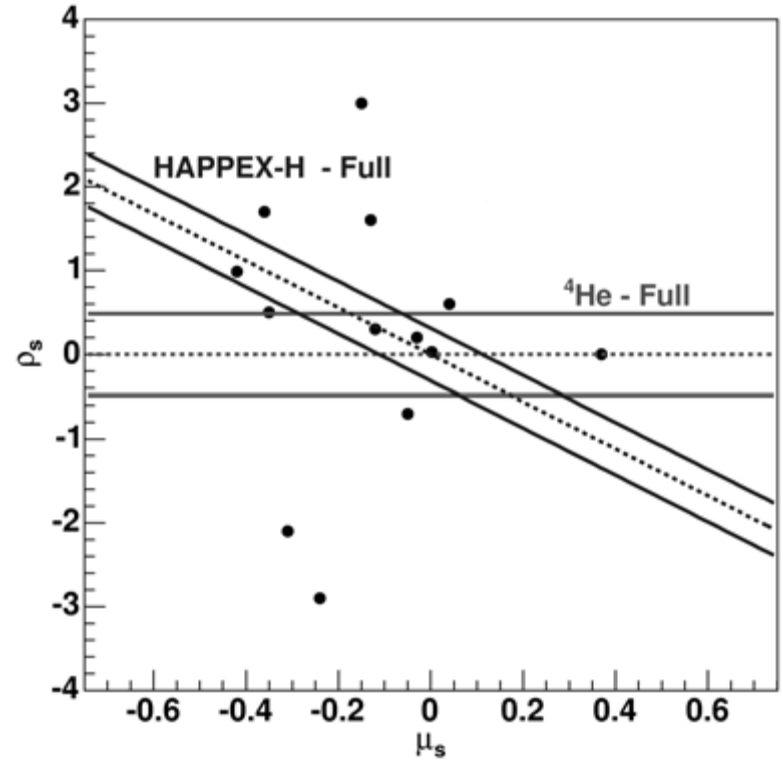

Fig. 2. Expected impact of HAPPEX-H (diagonal band) and HAPPEX-He (horizontal band). Points are predictions of various theoretical models. Expected limits on $\rho_{s}$ and $\mu_{s}$ are computed assuming the full data set and an extrapolation to $Q^{2}=0$, and are centered on $\rho_{s}=0, \mu_{s}=0$

been unacceptable for parity experiments. The new "racetrack" design features fluid flow transverse to the beam direction to reduce such boiling behavior.

For PREX, a thin lead target able to withstand beam intensity of tens of $\mu \mathrm{A}$ without melting is obviously a challenge. A design in which the foil is sandwiched between thin diamond films in a liquid helium cooled copper frame has been successfully tested. Diamond has high thermal conductivity and scattering from ${ }^{12} \mathrm{C}$ is well understood, making this an ideal solution for this experiment.

\section{Summary and prospects}

HAPPEX demonstrated the need for an experimental program to provide information on the electric and magnetic strange form factors separately, with high precision, at low $Q^{2}$. HAPPEX and HAPPEX-He are designed to significantly limit the space of allowed values for these strange quark effects. These are challenging experiments, but feasible with the improvements in equipment and techniques developed in the past several years. The expected impact is shown in Fig. 2. PREX is even more challenging, but we are confident our experience in HAPPEX-H and HAPPEX-He will establish our readiness to undertake it. HAPPEX-H and HAPPEX-He began data taking in Summer 2004, and preliminary results for both have been presented [18]. We expect to complete data taking for these experiments in 2005, with PREX to be scheduled subsequently.

\section{References}

1. J. Ashman et al.: Phys. Lett. B 206, 364 (1988); Nucl. Phys. B 328, 527 (1988); Nucl. Phys. B 328, 1 (1989)

2. D.B. Kaplan, A. Manohar: Nucl. Phys. B 310, 527 (1988)

3. R.D. McKeown: Phys. Lett. B 219, 140 (1989)

4. E.J. Beise, R.D. McKeown: Comments Nucl. Part. Phys. 20, 105 (1991)

5. D.H. Beck: Phys. Rev. D 39, 3248 (1989)

6. K. Aniol et al. [HAPPEX Collaboration]: Phys. Rev. Lett. 82, 1096 (1999); K. Aniol et al., [HAPPEX Collaboration]: Phys. Lett. B 509, 211 (2001)

7. K. Aniol et al. [HAPPEX Collaboration]: Phys. Rev. C 69, 065501 (2004)

8. T.M. Ito et al. [SAMPLE Collaboration]: Phys. Rev. Lett. 92, 102003 (2004), and references therein

9. F.E. Maas et al. [A4 Collaboration]: Phys. Rev. Lett. 93, 022002 (2004)

10. C.J. Horowitz, S.J. Pollock, P.A. Souder, R. Michaels: Phys. Rev. C 63, 025501 (2001)

11. R.M. Barnet et al. [Particle Data Group]: Phys. Rev. D 54, 1 (1996)

12. R. De Leo et al. [HAPPEX Collaboration]. JLab Experiment E99-115 Proposal (1999)

13. P.A. Souder et al. [HAPPEX Collaboration]: JLab Experiment E00-114 Proposal (2000)

14. K.A. Aniol et al. [HAPPEX Collaboration]: JLab Experiment E00-003 Proposal (1999); K.A. Aniol et al., [HAPPEX Collaboration]: JLab Experiment E03-011 Proposal Update (2002)

15. J. Alcorn et al.: Nucl. Instrum. Meth. A 522, 294 (2004)

16. A.V. Glamazdin et al.: Fizika B 8, 91 (1999)

17. M. Baylac, et al.: Phys. Lett. B 539, 8 (2002)

18. K. Paschke: Baryons 2004 conference (2004) 